\title{
Interactions and thermoelectric effects in a parallel-coupled double quantum dot
}

\author{
Miguel A. Sierra, ${ }^{1}$ M. Saiz-Bretín, ${ }^{2,3}$ F. Domínguez-Adame, ${ }^{2,3}$ and David Sánchez ${ }^{1}$ \\ ${ }^{1}$ Instituto de Física Interdisciplinar y Sistemas Complejos IFISC (UIB-CSIC), E-07122 Palma de Mallorca, Spain \\ ${ }^{2}$ GISC, Departamento de Física de Materiales, Universidad Complutense, E-28040 Madrid, Spain \\ ${ }^{3}$ Department of Physics, University of Warwick, Coventry CV4 7AL, United Kingdom \\ (Received 5 April 2016; revised manuscript received 7 June 2016; published 30 June 2016)
}

\begin{abstract}
We investigate the nonequilibrium transport properties of a double quantum-dot system connected in parallel to two leads, including intradot electron-electron interaction. In the absence of interactions, the system supports a bound state in the continuum. This state is revealed as a Fano antiresonance in the transmission when the energy levels of the dots are detuned. Using the Keldysh nonequilibrium Green's-function formalism, we find that the occurrence of the Fano antiresonance survives in the presence of Coulomb repulsion. We give precise predictions for the experimental detection of bound states in the continuum. First, we calculate the differential conductance as a function of the applied voltage and the dot level detuning and find that crossing points in the diamond structure are revealed as minima due to the transmission antiresonances. Second, we determine the thermoelectric current in response to an applied temperature bias. In the linear regime, quantum interference gives rise to sharp peaks in the thermoelectric conductance. Remarkably, we find interaction-induced strong current nonlinearities for large thermal gradients that may lead to several nontrivial zeros in the thermocurrent. The latter property is especially attractive for thermoelectric applications.
\end{abstract}

DOI: 10.1103/PhysRevB.93.235452

\section{INTRODUCTION}

Double quantum dots (DQDs) coupled in parallel serve as an excellent platform to test interaction and interference effects [1-3] because, due to the small system size, Coulomb repulsion is intensified and quantum phase coherence is preserved during an electron transfer between the attached reservoirs. Destructive interference between different paths traversing the two dots can lead to Fano resonances [4-6] and Dicke effect in the presence of a magnetic flux [7]. The interference pattern can be ultimately influenced by Coulomb interactions [8-11]. In the case of strong correlations, competition may arise between magnetic interactions and Kondo effect [12,13], Kondo states with higher symmetry [14,15], and signatures of quantum phase transitions [16]. Furthermore, these systems are interesting for solid-state quantum bit implementations and as probes of entanglement $[17,18]$.

Consider, for the moment, the case without interactions. The Fano effect originates from the quantum interference between resonant and nonresonant processes [19], giving rise to asymmetric electric conductance profiles in the nanostructure [20]. The occurrence of Fano antiresonances in DQD systems traces back to bound states in the continuum (BIC), formerly introduced in quantum mechanics by von Neumann and Wigner in 1929 as squared-integrable solutions buried in the continuum energy spectrum [21]. In the absence of interdot tunnel coupling, the local density of states at the quantum dots presents a narrow peak that approaches a $\delta$ function when the coupling to the leads vanishes, indicating that the resonant level becomes a true BIC [22,23]. It should be stressed that the interdot tunneling should be kept as small as possible to detect the occurrence of BICs in nonequilibrium transport experiments [23]. In this work, we would first like to analyze to what extent BICs survive in the presence of a Coulomb interaction. This is a natural question since, in experiments, small dots exhibit Coulomb blockade effects due to a finite on-site charging energy, which is typically the largest energy scale of the problem. Below we demonstrate that BICs are robust against charging effects and that, in fact, a replica of the original BIC emerges at high energies due to Coulomb repulsion.

The second objective of this paper is to address the question of whether parallel-coupled DQDs are useful in thermoelectrics. The problem has thus far attracted little attention [24,25] despite the fact that sharp resonances (such as the Fano line shapes) are, in principle, ideal candidates for highly efficient waste heat-to-electricity converters [26]. The issue can be viewed within the broader perspective of nanostructures as key elements in future thermoelectric applications based on the Seebeck effect. Such devices have many attractive features compared with other methods due to the absence of moving parts, scalability, and high reliability [27]. Closely connected with many discoveries that have demonstrated that nanometer-sized objects exhibit physical properties not shared by bulk materials [28], theoretical predictions [29-31] and experiments [32-35] have pointed out that thermoelectric properties at the nanoscale can be strongly enhanced. In fact, an enhancement of the thermoelectric figure of merit can be achieved with the aid of quantum interference phenomena [36-38]. In particular, quantum effects giving rise to Fano antiresonances in the transmission probability of DQD artificial molecules lead to departures from the Wiedemann-Franz law $[39,40]$. As a consequence, the thermoelectric efficiency is greatly enhanced [41]. While Refs. [24,25] neglect the role of BICs and focus on the linear regime of transport, here we find that the generated thermocurrent in response to an applied temperature bias can be large for small thermal gradients due to the important role of BICs in the DQD spectral density. More importantly, we obtain strongly nonlinear current-temperature characteristics that present different nontrivial zeros with enhanced peakto-valley ratios. This is an appealing functionality that relies on the electron-electron interaction in the DQD system and disappears in the noninteracting limit. 
Our work is structured as follows. In Sec. II, we discuss our theoretical model for a parallel-coupled DQD system with negligible interdot tunneling, but finite intradot Coulomb interaction. We calculate the electric current using the Keldysh nonequilibrium Green's-function formalism [42]. Specifically, we consider the Coulomb blockade regime and disregard cotunneling and Kondo correlations within an equation-ofmotion (EOM) technique. In Sec. III, we examine the spectral function and explicitly show the emergence of a BIC as a function of the detuning of the dot levels and the couplings between the dots and the leads. Here we compare the cases with and without electron-electron interactions. The transmission probability is discussed in Sec. IV. Since current can be driven by either electric or thermal means, we first treat the voltage transport in Sec. V and then the thermoelectric transport in Sec. VI. In both cases, we consider the linear conductances and the nonlinear regime of transport. Finally, Sec. VII summarizes our conclusions.

\section{MODEL AND FORMALISM}

We consider two quantum dots forming a DQD system, connected to left (L) and right (R) leads by tunnel couplings, as shown schematically in Fig. 1. Only one energy level in each dot is assumed relevant and electron-phonon interaction is neglected hereafter. However, on-site Coulomb interaction is considered for each dot.

\section{A. System Hamiltonian}

The Hamiltonian associated with the whole system can be written as $\mathcal{H}=\mathcal{H}_{\text {DQD }}+\mathcal{H}_{\text {leads }}+\mathcal{H}_{\text {tunnel }}$. Here, $\mathcal{H}_{\text {DQD }}$ describes the dynamics of interacting electrons in the DQD system,

$$
\mathcal{H}_{\mathrm{DQD}}=\sum_{i \sigma} \varepsilon_{i} n_{i \sigma}+\sum_{i} U_{i} n_{i \uparrow} n_{i \downarrow},
$$

where the index $i=1,2$ runs over the quantum dots and $U_{i}$ is the Coulomb energy when the dots are doubly occupied. The number operator is $n_{i \sigma}=d_{i \sigma}^{\dagger} d_{i \sigma}$, where $d_{i \sigma}^{\dagger}\left(d_{i \sigma}\right)$ is the creation (annihilation) operator of an electron in the dot $i$ with energy $\varepsilon_{i}$ and spin $\sigma$. Quantum dots are assumed to be far apart so tunnel and capacitive couplings between them are weak. This weakness facilitates the possibility of detecting the BICs in transport experiments [23]. Electrons in the ideal leads are

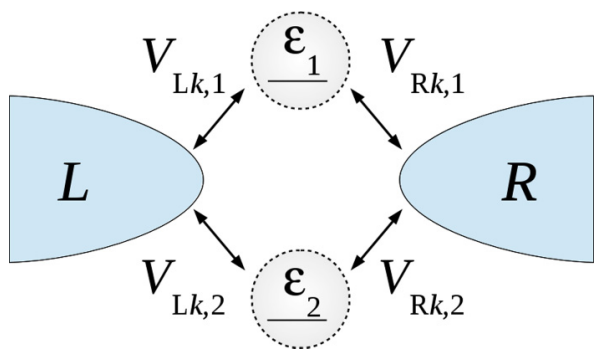

FIG. 1. Schematic diagram of the DQD system, connected to leads $L$ and $R$. We indicate the energy levels $\varepsilon_{1}$ and $\varepsilon_{2}$, and the tunnel amplitudes $V_{L k, 1}, V_{L k, 2}, V_{R k, 1}$, and $V_{R k, 2}$. We take into account intradot electron-electron interactions (not shown here). regarded as noninteracting particles with crystal momentum $k$ and energy $\varepsilon_{\alpha k \sigma}$. The corresponding Hamiltonian is

$$
\mathcal{H}_{\text {leads }}=\sum_{\alpha k \sigma} \varepsilon_{\alpha k \sigma} C_{\alpha k \sigma}^{\dagger} C_{\alpha k \sigma} .
$$

Here, $C_{\alpha k \sigma}^{\dagger}\left(C_{\alpha k \sigma}\right)$ denotes the creation (annihilation) operator of a conduction electron in the semi-infinite lead, $\alpha=L, R$. Finally, the quantum dots are tunnel coupled to both leads (see Fig. 1). Hence,

$$
\mathcal{H}_{\text {tunnel }}=\sum_{\alpha k \sigma i} V_{\alpha k, i} C_{\alpha k \sigma}^{\dagger} d_{i \sigma}+\text { H.c. }
$$

where H.c. stands for Hermitian conjugate and the amplitudes $V_{\alpha k, i}$ are tunnel coupling parameters. These factors are spin independent for nonmagnetic leads.

\section{B. Charge current}

The charge current is obtained as the time derivative of the expected occupation in one of the leads, $I_{\alpha}=-e d\left\langle n_{\alpha}(t)\right\rangle / d t$ with $n_{\alpha}(t)=\sum_{k \sigma} C_{\alpha k \sigma}^{\dagger}(t) C_{\alpha k \sigma}(t)$. In the steady-state case, charge conservation demands that $I_{L}+I_{R}=0$. Hence we can define the charge current flowing through the DQD system as $I \equiv I_{L}=-I_{R}$. Following Ref. [42], the charge current can be cast in the form (we set $\hbar=1$ hereafter)

$$
I=\frac{e}{\pi} \int_{-\infty}^{\infty} d E \sum_{k \sigma i} \operatorname{Re}\left[V_{\alpha k, i} G_{i \sigma, \alpha k \sigma}^{<}(E)\right],
$$

where $G_{i \sigma, \alpha k \sigma}^{<}(E)$ is the Fourier transform of the lesser Green's function $G_{i \sigma, \alpha k \sigma}^{<}(t) \equiv i\left\langle C_{\alpha k \sigma}^{\dagger}(0) d_{i \sigma}(t)\right\rangle$.

We now define the parameters $\Gamma_{i j}^{\alpha}=2 \pi \rho_{\alpha} V_{\alpha k, i} V_{\alpha k, j}^{*}$, with $\rho_{\alpha}$ being the density of states of the lead $\alpha$. In the wideband limit, these parameters are assumed to be independent of the electron energy. As a consequence, the numbers $\Gamma_{i j}^{\alpha}$ are constants. Langreth rules [42] allow the charge current to be expressed solely in terms of the advanced and retarded Green's functions of the dots,

$$
\begin{aligned}
I= & \frac{e}{2 \pi} \sum_{\sigma} \int_{-\infty}^{\infty} d E\left[f_{L}\left(E, T_{L}\right)-f_{R}\left(E, T_{R}\right)\right] \\
& \times \operatorname{Tr}\left[\boldsymbol{G}_{\sigma \sigma}^{a}(E) \boldsymbol{\Gamma}^{R} \boldsymbol{G}_{\sigma \sigma}^{r}(E) \boldsymbol{\Gamma}^{L}\right]
\end{aligned}
$$

where $f_{\alpha}\left(E, T_{\alpha}\right)=\left\{\exp \left[\left(E-\mu_{\alpha}\right) / k_{B} T_{\alpha}\right]+1\right\}^{-1}$ is the Fermi distribution function of the lead $\alpha$ with electrochemical potentials $\mu_{L}=\varepsilon_{F}+e V / 2$ and $\mu_{R}=\varepsilon_{F}-e V / 2$ and temperatures $T_{L}$ and $T_{R}$. The matrix elements of $\Gamma^{\alpha}$ are given by $\Gamma_{i j}^{\alpha}$. In this expression, $\boldsymbol{G}_{\sigma \sigma}^{r}(E)$ is a $2 \times 2$ matrix whose elements are the Fourier transform of the retarded Green's functions,

$$
G_{i \sigma, j \sigma}^{r}(t)=-i \theta(t)\left\langle\left[d_{i \sigma}(t), d_{j \sigma}^{\dagger}(0)\right]_{+}\right\rangle,
$$

where $\theta$ is the Heaviside step function and $[\cdot]_{+}$stands for the anticommutator. Similarly, $G_{i \sigma, j \sigma}^{a}(E)$ is the Fourier transform of the advanced Green's functions $G_{i \sigma, j \sigma}^{a}(t)=$ $i \theta(-t)\left\langle\left[d_{i \sigma}(t), d_{j \sigma}^{\dagger}(0)\right]_{+}\right\rangle$. 


\section{Equation-of-motion approach}

We restrict ourselves to the Coulomb-blockade regime, for which the Coulomb energy $U_{i}$ is assumed to be much larger than the background temperature $k_{B} T$ and the hybridization parameters $\Gamma_{i j}^{\alpha}$. Using the EOM method, the retarded Green's functions can be assessed by neglecting the correlators $\left\langle\left\langle d_{i \sigma} d_{i \bar{\sigma}}^{\dagger} C_{\alpha k \bar{\sigma}}, d_{j \sigma}\right\rangle\right\rangle_{t} \simeq 0$ and $\left\langle\left\langle d_{i \sigma} C_{\alpha k \bar{\sigma}}^{\dagger} d_{i \bar{\sigma}}, d_{j \sigma}^{\dagger}\right\rangle\right\rangle_{t} \simeq$ 0 , corresponding to virtual charge excitations of the dots. This approach holds for strongly interacting quantum dots at temperatures above the Kondo temperature. We have introduced the notation $\langle\langle A, B\rangle\rangle_{t}=i \theta(t)\left\langle[A(t), B(0)]_{+}\right\rangle$for brevity. After straightforward algebra, we obtain the following equations for the Fourier transforms of the Green's functions and correlators:

$$
\begin{aligned}
& \left(E-\varepsilon_{i}\right) G_{i \sigma, j \sigma}^{r} \\
& \quad=\delta_{i j}+U_{i}\left\langle\left\langle d_{i \sigma} n_{i \bar{\sigma}}, d_{i \sigma}^{\dagger}\right\rangle\right\rangle+\sum_{\alpha k} V_{\alpha k, i}^{*} G_{\alpha k \sigma, j \sigma}^{r}, \\
& \left(E-\varepsilon_{i}-U_{i}\right)\left\langle\left\langle d_{i \sigma} n_{i \bar{\sigma}}, d_{j \sigma}^{\dagger}\right\rangle\right\rangle \\
& \quad=\left\langle n_{i \bar{\sigma}}\right\rangle \delta_{i j}+\sum_{\alpha k} V_{\alpha k, i}^{*}\left\langle\left\langle C_{\alpha k \sigma} n_{i \bar{\sigma}}, d_{j \sigma}^{\dagger}\right\rangle,\right.
\end{aligned}
$$

where $\langle\langle A, B\rangle\rangle$ denotes the Fourier transform of $\langle\langle A, B\rangle\rangle_{t}$. Hereafter, we consider the decoupling given by the Hubbard I approximation, $\left\langle\left\langle C_{\alpha k \sigma} n_{i \bar{\sigma}}, d_{i \sigma}^{\dagger}\right\rangle\right\rangle \approx\left\langle n_{i \bar{\sigma}}\right\rangle G_{\alpha k \sigma, i \sigma}^{r}$ [43]. Equations (4) are then readily solved, yielding a closed expression for the retarded Green's functions,

$$
\begin{aligned}
G_{i \sigma, j \sigma}^{r}(E)= & h_{i j}(E)\left\{\frac{1-\left\langle n_{i \bar{\sigma}}\right\rangle}{E-\varepsilon_{i}-\left(1+\frac{\left\langle n_{i \bar{\sigma}}\right\rangle U_{i}}{E-\varepsilon_{i}-U_{i}}\right) \widetilde{\Sigma}_{i}(E)}\right. \\
& \left.+\frac{\left\langle n_{i \bar{\sigma}}\right\rangle}{E-\varepsilon_{i}-U_{i}-\left[1-\frac{\left(1-\left\langle n_{i \bar{\sigma}}\right\rangle\right) U_{i}}{E-\varepsilon_{i}}\right] \widetilde{\Sigma}_{i}(E)}\right\} .
\end{aligned}
$$

For the sake of brevity, we have introduced the following definitions:

$$
\begin{gathered}
\Delta n_{i}=1+\frac{U_{i}}{E-\varepsilon_{i}-U_{i}}\left\langle n_{i \bar{\sigma}}\right\rangle, \\
\widetilde{\Sigma}_{i}(E)=\Sigma_{i i}+\frac{\Sigma_{\bar{i} i} \Sigma_{i \bar{i}}}{E-\varepsilon_{\bar{i}}-\Delta n_{\bar{i}} \Sigma_{\bar{i} \bar{i}}} \Delta n_{\bar{i}}, \\
h_{i j}(E)=\delta_{i j}+\frac{\Delta n_{\bar{i}} \Sigma_{\bar{i} i}}{E-\varepsilon_{\bar{i}}-\Delta n_{\bar{i}} \Sigma_{\bar{i} \bar{i}}} \delta_{\bar{i} j} .
\end{gathered}
$$

Within the wideband limit, the self-energy becomes $\Sigma_{i j}=$ $-i\left(\Gamma_{i j}^{L}+\Gamma_{i j}^{R}\right) / 2 \equiv-i \Gamma_{i j} / 2$. According to Eq. (5a), the diagonal elements $G_{i \sigma, i \sigma}^{r}(E)$ have two poles that are shifted (with respect to the bare values $E=\varepsilon_{i}$ and $E=\varepsilon_{i}+U_{i}$ ) and broadened due to the presence of the complex term $\widetilde{\Sigma}_{i}$. In the case of weak interdot capacitive interaction, the Hamiltonian given by Eq. (1a) acquires a term $U_{12} N_{1} N_{2}$, where $N_{i}=\sum_{\sigma} n_{i \sigma}$, and this term can be treated within the Hartree approximation. As a consequence, the two poles found above would become renormalized due to an additional factor $-U_{12}\left\langle N_{\bar{l}}\right\rangle$ in the denominator of Eq. (5a). However, our results will qualitatively remain unaffected.
The tunnel coupling of the DQD system to both leads is encoded in the matrices [44]

$$
\Gamma^{L}=\Gamma_{0}\left(\begin{array}{cc}
1 & \sqrt{a} \\
\sqrt{a} & a
\end{array}\right), \quad \Gamma^{R}=\Gamma_{0}\left(\begin{array}{cc}
a & \sqrt{a} \\
\sqrt{a} & 1
\end{array}\right) .
$$

Here, $\Gamma_{0}$ and $a$ are parameters describing the different coupling of each dot to both leads. This coupling corresponds to the configuration studied in Ref. [4]. For instance, $a=0$ corresponds to totally decoupled quantum dots and $a=1$ to a symmetric coupling case. The spin-dependent occupations $\left\langle n_{i \bar{\sigma}}\right\rangle$ can be calculated from the general expression

$$
\left\langle d_{i \bar{\sigma}}^{\dagger} d_{j \bar{\sigma}}\right\rangle=\int \frac{d E}{2 \pi i} G_{j \bar{\sigma}, i \bar{\sigma}}^{<}(E) .
$$

The lesser Green's function is related to the retarded and advanced Green's functions as $\boldsymbol{G}_{\sigma, \sigma}^{<}(E)=$ $i \sum_{\alpha} f_{\alpha}(E) \boldsymbol{G}_{\sigma, \sigma}^{r} \boldsymbol{\Gamma}^{\alpha} \boldsymbol{G}_{\sigma, \sigma}^{a}$. Therefore,

$$
\left\langle n_{i \bar{\sigma}}\right\rangle=\int \frac{d E}{2 \pi} \sum_{\alpha l m} f_{\alpha}(E) G_{i \bar{\sigma}, l \bar{\sigma}}^{r}(E) \Gamma_{l m}^{\alpha} G_{m \bar{\sigma}, i \bar{\sigma}}^{a}(E) .
$$

Equations (5a) and (7b) are solved self-consistently to obtain $G_{i \sigma, j \sigma}^{r}(E)$.

\section{SPECTRAL FUNCTION}

The spectral function per spin is defined as

$$
\mathcal{A}(E)=-\frac{1}{\pi} \operatorname{Im} \operatorname{Tr} \mathbf{G}_{\sigma \sigma}^{r}(E) .
$$

The absence of magnetic interactions ensures that $\mathcal{A}(E)$ becomes spin independent. In this section, we discuss the spectral function in equilibrium at $T=0$ for concreteness $\left(\mu_{L}=\mu_{R}=0\right.$ and $\left.T_{L}=T_{R}=0\right)$.

To gain insight into the occurrence of BICs in the DQD system, first we consider the noninteracting case by setting $U_{i}=0$ for the moment. We take $\varepsilon_{1}=-\varepsilon_{2} \equiv \varepsilon$ to obtain simpler expressions, although more general situations can be handled in the same way. A lengthy but straightforward calculation yields the following expression for the spectral density in the noninteracting case:

$$
\begin{aligned}
\mathcal{A}(E)= & \frac{(1+a) \Gamma_{0}}{\pi D(E)}\left[E^{2}+\varepsilon^{2}+\frac{1}{4}(1-a)^{2} \Gamma_{0}^{2}\right], \\
D(E) \equiv & \left(E^{2}-\varepsilon^{2}\right)^{2}+\left[\frac{\Gamma_{0}}{2}(1-a)\right]^{4} \\
& +\frac{\Gamma_{0}^{2}}{2}\left[E^{2}\left(1+6 a+a^{2}\right)+\varepsilon^{2}(1-a)^{2}\right] .
\end{aligned}
$$

Taking $a=1$ (symmetric coupling to leads) and $|E|<\varepsilon<$ $\Gamma_{0}$, the spectral function is approximately given as

$$
\mathcal{A}(E) \simeq \frac{1}{\pi} \frac{2 \Gamma_{0}}{E^{2}+4 \Gamma_{0}^{2}}+\frac{1}{\pi} \frac{\varepsilon^{2} / 2 \Gamma_{0}}{E^{2}+\left(\varepsilon^{2} / 2 \Gamma_{0}\right)^{2}} .
$$

The spectral function is the sum of two Lorentzians centered at $E=0$, originated from the superposition of two states. One of these states is strongly coupled to the continuum, giving rise to a wide peak of width $2 \Gamma_{0}$. However, the level broadening of the other state is small when $\varepsilon<\Gamma_{0}$, indicating that it is only weakly coupled to the continuum. Tuning the levels of both dots at resonance $(\varepsilon \rightarrow 0)$, the spectral function 
becomes

$$
\mathcal{A}(E) \simeq \frac{1}{\pi} \frac{2 \Gamma_{0}}{E^{2}+4 \Gamma_{0}^{2}}+\delta(E) .
$$

The presence of a $\delta$ function in $\mathcal{A}(E)$ characterizes a truly bound state at $E=0$. This localized state becomes effectively decoupled from the continuum states when $a \rightarrow 1$ and $\varepsilon_{1} \rightarrow$ $\varepsilon_{2}$, but its energy lies at the band center. Therefore, the DQD system with negligible Coulomb interaction supports a BIC $[22,23]$. It should be stressed that nonsymmetric tunnel coupling to the leads $(a \neq 1)$ smears out the BIC and only a single peak of finite width arises when $\varepsilon=0$. From Eq. (9a), one finds, in this case,

$$
\mathcal{A}(E) \sim \frac{1}{\pi} \frac{\gamma(a)}{E^{2}+\gamma^{2}(a)},
$$

if $|E|<\Gamma_{0}$ [23]. The width of the Lorentzian profile $\gamma(a) \equiv$ $\Gamma_{0}(1-a)^{2} / \sqrt{8\left(1+6 a+a^{2}\right)}$ only vanishes in the limit $a \rightarrow$ 1 , as expected.

We now turn to one of our main goals, namely, the effects of the Coulomb interaction on the BICs discussed above. At finite values of the Coulomb energy $U_{i}$, Eqs. (5a) and (7b) have to be solved numerically. Figure 2 shows with a solid line the spectral function $\mathcal{A}(E)$ at $T=0$ when $U_{1}=U_{2} \equiv U=1$. Hereafter, we take $\Gamma_{0}=1$ as our unit of energy. Results for the noninteracting DQD system $(U=0)$ are shown as a dashed line for comparison. According to Eq. (9b), the spectral function of the noninteracting DQD system $(U=0)$ is a superposition of two Lorentzians of finite width when the tunnel coupling to the leads is symmetric $(a=1)$ and the dots are detuned $(\varepsilon=1)$, as seen in blue in Fig. 2(a). The two Lorentzians cannot be resolved since their widths $2 \Gamma_{0}$ and $\varepsilon^{2} / 2 \Gamma_{0}$ do not differ much with the chosen parameters. After
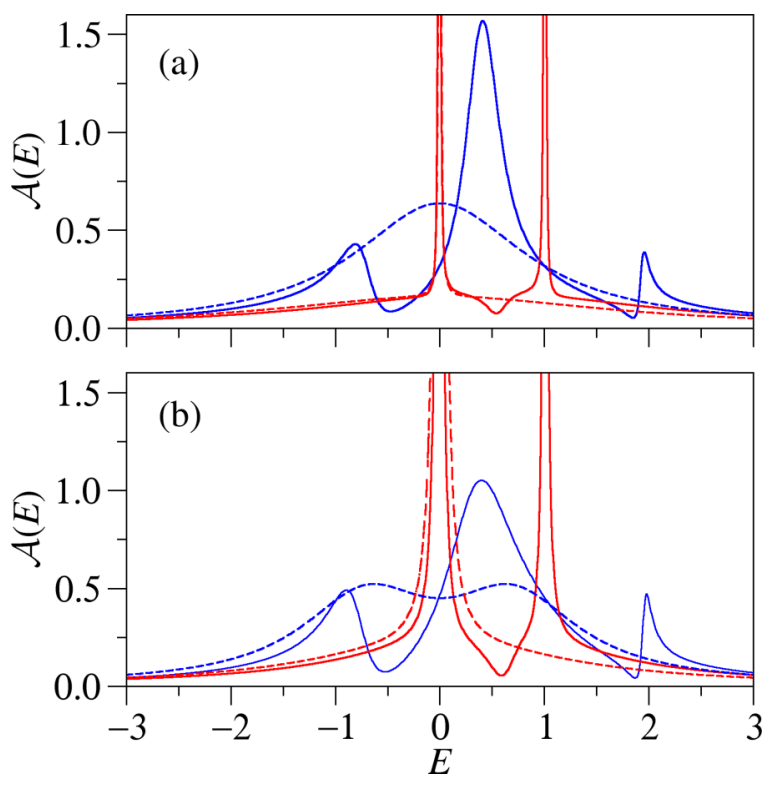

FIG. 2. Spectral function at $T=0$ for a DQD system tunnel coupled to leads (a) symmetrically $(a=1)$ and (b) nonsymmetrically $(a=0.5)$. Solid and dashed lines correspond to interacting $(U=1)$ and noninteracting $(U=0)$ electrons, respectively; meanwhile the color line is blue $(\varepsilon=1.0)$ or red $(\varepsilon=0.05)$. Energies are expressed in units of $\Gamma_{0}$. the Coulomb interaction is switched on, virtual levels around $\varepsilon_{i}+U$ arise, giving rise to a complex pattern with maxima and minima. Maxima and inflection points are located at energies $\pm \varepsilon$ and $\pm \varepsilon+U$.

More interesting results are found in the limit $\varepsilon \rightarrow 0$, shown in red in Fig. 2(a). In the noninteracting DQD system, the spectral function presents a narrow peak at $E=0$ when $\varepsilon$ is small but finite. This central peak resembles the $\delta$-function term in Eq. (9c) when $\varepsilon=0$ and signals the occurrence of a BIC. Most importantly, the peak is replicated at an energy $E=$ $U$ when Coulomb interaction is finite. The numerical evidence that the BIC is still present and replicated at $E=U$ can be substantiated by noticing that the occupation of both quantum dots is the same, $\left\langle n_{1 \bar{\sigma}}\right\rangle=\left\langle n_{2 \bar{\sigma}}\right\rangle \equiv\langle n\rangle$, when $\varepsilon=0$ and the coupling to the leads is symmetric ( $a=1)$. Proceeding in the same way as in Eq. (9d), the spectral function given by Eq. (8) can be calculated from the retarded Green's function (5a) in this limit case. Neglecting the contribution of the resonant states strongly coupled to the continuum, Eq. (5a) has two poles at energies around the resonances $\left(|E|,|E-U| \ll \Gamma_{0}\right)$, weighted by the occupations. We thus find, for the spectral function,

$$
\mathcal{A}(E) \simeq[1-\langle n\rangle] \delta(E)+\langle n\rangle \delta(E-U),
$$

in the limit $\varepsilon=0$, in perfect agreement with the numerics. As expected, the BIC replicated at $E=U$ vanishes when the occupation is negligible.

Hence, we come to the important conclusion that BICs are preserved even in the presence of the Coulomb interaction.

Figure 2(b) shows the spectral density in the case of asymmetric tunnel coupling to the leads $(a=0.5)$. When $U=0$ and $\varepsilon$ is large (detuned quantum dots), the spectral function is bimodal, according to the general expression (9a). Similarly to the case of symmetric coupling, more features arise when $U$ is finite. The two maxima merge into a single peak at $E=0$ when the levels of the dots approach each other $(\varepsilon \rightarrow 0)$ and this peak is replicated at $E=U$ when the Coulomb interaction is finite.

\section{TRANSMISSION COEFFICIENT}

The EOM approach introduced in Sec. II C turns the initial many-body problem into an effective one-body problem. In this section, we focus on the transmission function, given by Eq. (11), which enters the current formula, given by Eq. (2b). Under this premise, the transmission coefficient can be expressed as [45]

$$
\tau(E)=\operatorname{Tr}\left[\boldsymbol{G}_{\sigma \sigma}^{a}(E) \boldsymbol{\Gamma}^{R} \boldsymbol{G}_{\sigma \sigma}^{r}(E) \boldsymbol{\Gamma}^{L}\right] .
$$

We restrict ourselves to equilibrium by setting $\mu_{L}=\mu_{R}=0$ and $T_{L}=T_{R}=0$ throughout this section. In the absence of Coulomb interaction, the transmission coefficient can be obtained analytically: $\tau(E)=4 a \Gamma_{0}^{2} E^{2} / D(E)$ [23], with $D(E)$ given by Eq. (9b). When $D(E=0) \neq 0$, the transmission coefficient vanishes at $E=0$. For instance, when the coupling to the leads is symmetric $(a=1)$ but the energy levels of the dots are detuned $(\varepsilon \neq 0)$, one obtains

$$
\tau(E) \simeq \frac{E^{2}}{E^{2}+\varepsilon^{4} / 4 \Gamma_{0}^{2}},
$$



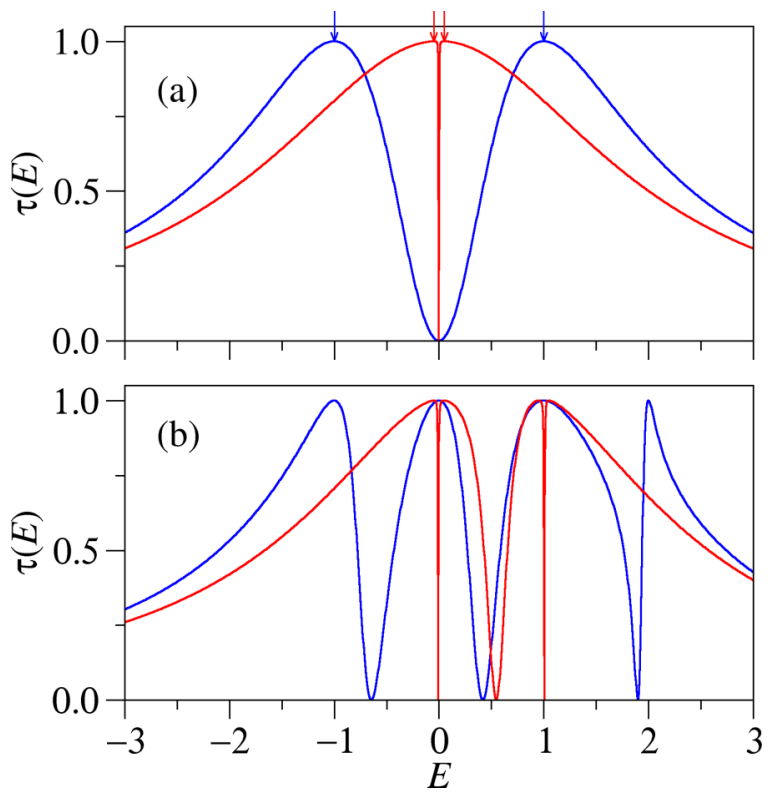

FIG. 3. Transmission coefficient at $T=0$ for a DQD system tunnel coupled symmetrically to leads $(a=1)$, corresponding to (a) noninteracting $(U=0)$ and (b) interacting $(U=1)$ electrons, respectively. Color line is blue $(\varepsilon=1.0)$ or red $(\varepsilon=0.05)$. Blue and red arrows in the upper panel indicate $E= \pm \varepsilon$. Energies are expressed in units of $\Gamma_{0}$.

for $|E|<\varepsilon$. The transmission coefficient becomes unity at energies $\pm \varepsilon$, as indicated by vertical arrows in Fig. 3(a). We remark that the transmission coefficient displays a Fano antiresonance profile of width $\varepsilon^{2} / 2 \Gamma_{0}$, originated from the interference of two coexisting paths for a traveling electron in the system. One path is a direct way that traverses the DQD via the strongly coupled state, while the second path includes a hopping on and off the BIC and then the electron continues with propagation. The destructive interference between these two paths is at the heart of the Fano antiresonance, as shown in Eq. (12a) [46].

Similarly, when the energy levels are aligned $(\varepsilon=0)$ but the coupling to the leads is asymmetric $(a \neq 1)$, the transmission again shows a Fano antiresonance around $E=0$, namely $\tau(E) \sim E^{2} /\left[E^{2}+\gamma^{2}(a)\right]$. Although transmission may take large values at some specific energies if $a \neq 1$, it should be mentioned that the DQD system never reaches perfect transparency.

From Eq. (12a), it becomes apparent that the dip gets narrower when the levels of the quantum dots approach each other, as seen in Fig. 3(a) for $\varepsilon=0.05$ (red curve), and eventually disappears if $\varepsilon=0$. In this limit case, corresponding to $D(E=0) \rightarrow 0$ when $a=1$, the transmission coefficient displays a Lorentzian shape of width $2 \Gamma_{0}$,

$$
\tau(E)=\frac{4 \Gamma_{0}^{2}}{E^{2}+4 \Gamma_{0}^{2}} .
$$

Therefore, unless the system is finely tuned $(\varepsilon=0$ and $a=1$ ), in general the transmission coefficient shows a marked $\operatorname{dip}$ at $E=0$.

For the interacting DQD system, the transmission coefficient given by Eq. (11) has to be computed numerically from Eqs. (5a) and (7b). In Fig. 3(b), we observe that the transmission coefficient at $E=0$ becomes unity when $\varepsilon=1$ (blue curve), whereas it vanishes if $U=0$ [see Fig. 3(a)]. Coulomb interaction splits the levels and the transmission coefficient, then displays four maxima at energies $E= \pm \varepsilon$ and $E= \pm \varepsilon+U$, as seen in Fig. 3(b). Moreover, in the limit $\varepsilon \rightarrow 0$, the narrow antiresonance at $E=0$ found when $U=0$ is replicated at $E=U$ in the interacting DQD system. This result is consistent with the spectral function discussed in the previous section and confirms the robustness of BICs under Coulomb interaction.

\section{VOLTAGE-DRIVEN ELECTRIC TRANSPORT}

We now discuss the impact of BICs on the electric response of the DQD system out of equilibrium. To this end, we calculate the nonlinear dependence of the electric current given by Eq. (2b) on the source-drain voltage $V$. Crossing of the four levels involved, namely $\varepsilon_{i}$ and $\varepsilon_{i}+U_{i}$, makes the Fano antiresonance in the transmission narrower and eventually it disappears when the system is finely tuned, as discussed in the previous section. The crossing reveals itself as minima of the differential conductance curves $G=d I / d V$, as seen in Fig. 4 (here we set the Fermi energy $\varepsilon_{F}=0$ and the rest of the energies in units of $\Gamma_{0}$ ). Figure 4(a) displays the low-temperature differential conductance as a function of $e V$ and $\varepsilon_{2}$ for $\varepsilon_{1}=2, U_{1}=U_{2}=3$, and $T_{L}=T_{R}=T=10^{-3}$. We observe that the differential conductance displays abrupt minima when the two levels simultaneously cross $e V / 2$ or $-e V / 2$. This condition is better seen in Fig. 4(b), where we plot the eight straight lines corresponding to the resonant conditions $e V= \pm 2 \varepsilon_{i}$ and $e V= \pm 2\left(\varepsilon_{i}+U_{i}\right)$. It is worth noting that similar conductance patterns are found for interacting twoorbital quantum dots [47]. Solid circles indicate the crossing points where the differential conductance reaches a local minimum, in perfect agreement with Fig. 4(a). Therefore, the existence of BICs can be detected in transport experiments by measuring the differential conductance. It should be mentioned that the differential conductance displays local maxima when one level crosses $e V / 2$ and the other reaches the value $-e V / 2$. These maxima are marked with open circles in Fig. 4(b).

\section{TEMPERATURE-DRIVEN ELECTRIC TRANSPORT}

We now investigate the electric transport in response to a thermal gradient $\theta$. For $\theta>0$, we take $T_{L}=T+\theta$ and $T_{R}=$ $T$ with $T$ the background temperature, while for $\theta<0$, we take $T_{L}=T$ and $T_{R}=T+|\theta|$. Thus, $\theta$ is positive (negative) when the left (right) electrode is the hotter contact. In this section, the bias voltage is absent $\left(\mu_{L}=\mu_{R}=\varepsilon_{F}\right.$ or $\left.V=0\right)$ and charge flows only due to the external thermal gradient. The differential thermoelectric conductance $L=d I / d \theta$ for $\theta>0$ is expressed as

$$
L=\frac{e}{h} \int_{-\infty}^{\infty} d E\left[g(E, \theta)+\left(f_{L}-f_{R}\right) \frac{\partial}{\partial \theta}\right] \tau(E, \theta),
$$

with

$$
g(E, \theta) \equiv \frac{E-\varepsilon_{F}}{T_{L}}\left[-\frac{\partial f_{L}(E, \theta)}{\partial E}\right],
$$



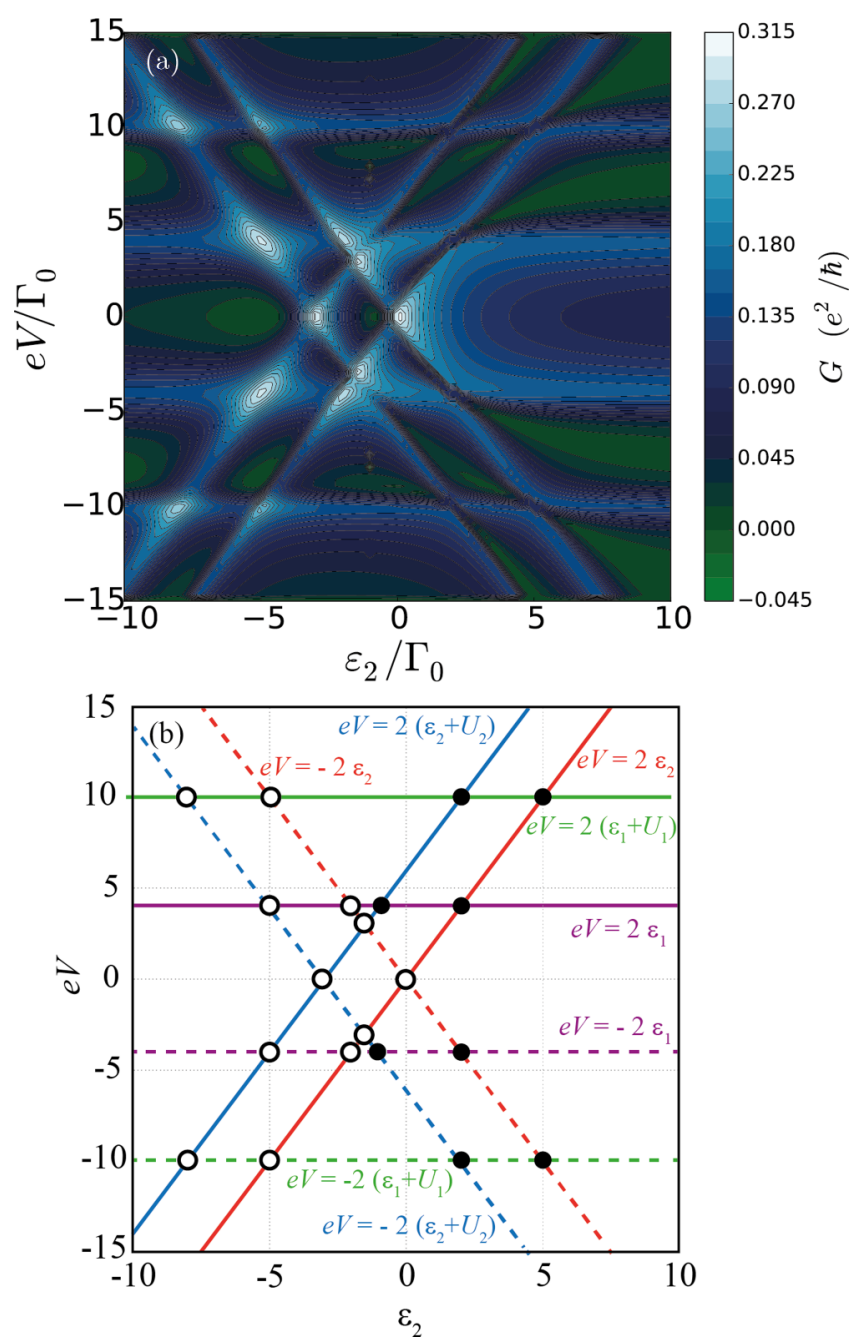

FIG. 4. (a) Differential electric conductance as a function of $e V$ and $\varepsilon_{2}$ for $\varepsilon_{1}=2, U_{1}=U_{2}=3$, and $T_{L}=T_{R}=T=10^{-3}$. (b) Diagram of the energy levels showing crossing points that correspond to maxima (open circles) and minima (solid circles) of the differential conductance. Energies are expressed in units of $\Gamma_{0}$.

and similarly for $\theta<0$ after making the substitutions $f_{L}(E, \theta) \leftrightarrow-f_{R}(E, \theta)$ and $T_{L} \leftrightarrow T_{R}$ in the first term of the integrand. Since $g(E, \theta)$ is an odd function of $E-\mu_{L}$, the thermoelectric conductance vanishes if the transmission coefficient is a symmetric function of the energy $E$ with respect to the Fermi level $\varepsilon_{F}$.

In Fig. 5, we depict the linear thermoelectric conductance $L_{0}$ of the DQD system as a function of the Fermi energy. $L_{0}$ is obtained from Eq. (13a) in the limit $\theta \rightarrow 0$. As a consequence, the second term in the right-hand side of Eq. (13a) cancels out and the functions $g$ and $\tau$ are evaluated at $\theta=0$. At low temperature, a Sommerfeld expansion shows that $L_{0}$ is proportional to the derivative of $\tau$ with respect to energy at $E=\varepsilon_{F}$ [48]. Therefore, $L_{0}$ is a magnitude sensitive to changes in the transmission, which makes it useful in the detection of narrow resonances.

Figure 5(a) shows the case $\varepsilon=0.05$. We observe two asymmetric resonances when $\varepsilon_{F}=0$ and $\varepsilon_{F}=1$ and a smooth variation around $\varepsilon_{F}=0.5$. We attribute the former to the
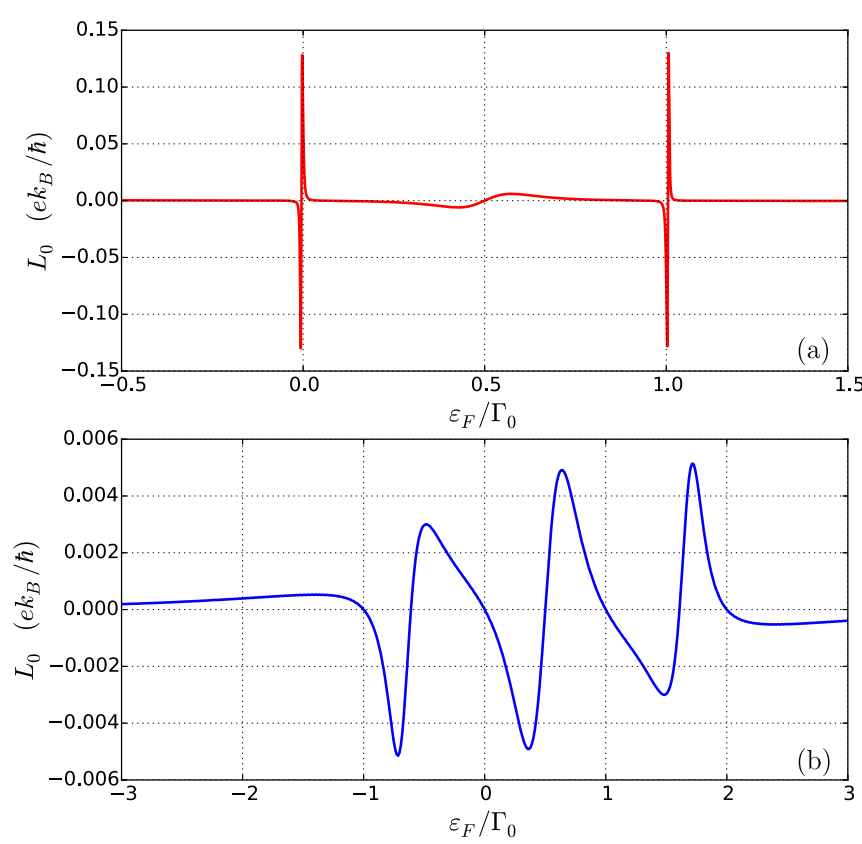

FIG. 5. Linear thermoconductance as a function of the temperature bias $k_{B} \theta$ for (a) $\varepsilon=0.05$ and (b) $\varepsilon=1$. The charging energies are $U_{1}=U_{2}=1$ and the background temperature is $k_{B} T=0.001$. Energies are expressed in units of $\Gamma_{0}$.

transmission dips seen in Fig. 3(b) and the latter to the more broadened antiresonance at $E=0.5$. However, one would expect that $L_{0}$ crosses zero whenever $\varepsilon_{F}$ aligns with an extremum of $\tau$ (maximum or minimum). The contribution of the transmission maxima cannot be observed in Fig. 5(a) due to the sharp variation around the dips. This can be demonstrated with a larger value of $\varepsilon[\varepsilon=1$ in Fig. 5(b)]. Both the maxima and the minima in $\tau$ now have comparable widths [see Fig. 3(b)] and their effect in $L_{0}$ is hence visible: There exist seven zeros in Fig. 5(b), which exactly correspond to the extrema of $\tau$. Importantly, $L_{0}$ reaches values as significant as 0.12 (in units of $e k_{B} / \hbar=20 \mathrm{nA} / \mathrm{K}$ ) for the case of the narrow antiresonances $(\varepsilon=0.05)$, showing that BICs can be useful in thermoelectric applications that convert waste heat into electricity.

Let us turn to the properties of the differential conductance given by Eq. (13a). In Ref. [49], two of us predicted the appearance of a butterfly structure in $L$ as a function of the applied temperature bias and the energy level of a single dot. This characteristic shape can ultimately be traced back to the presence of two resonances in the dot due to Coulomb repulsion, which has been confirmed experimentally [50]. In the case of the parallel DQD system considered here, we have quantum interference effects that distort the butterfly structure. In Fig. 6, we show $L$ as a function of $\theta$ and $\varepsilon_{2}$ for a fixed value $\varepsilon_{1}=2 \Gamma_{0}$. When $U_{1}=U_{2}=0$, we find a vanishing thermoelectric conductance for $\varepsilon_{2}=-2 \Gamma_{0}$ independently of the temperature bias [see Fig. 6(a)]. We recall that the configuration $\varepsilon_{1}=-\varepsilon_{2}$ leads to transmission functions that are symmetric relative to the Fermi energy [see Fig. 3(a)]. As a consequence, $L=0$ to all orders in $\theta$. Away from $\varepsilon_{2}=-2 \Gamma_{0}$, we can explain the behavior for small $\theta$ as follows. As $\varepsilon_{2}$ increases, the transmission function 

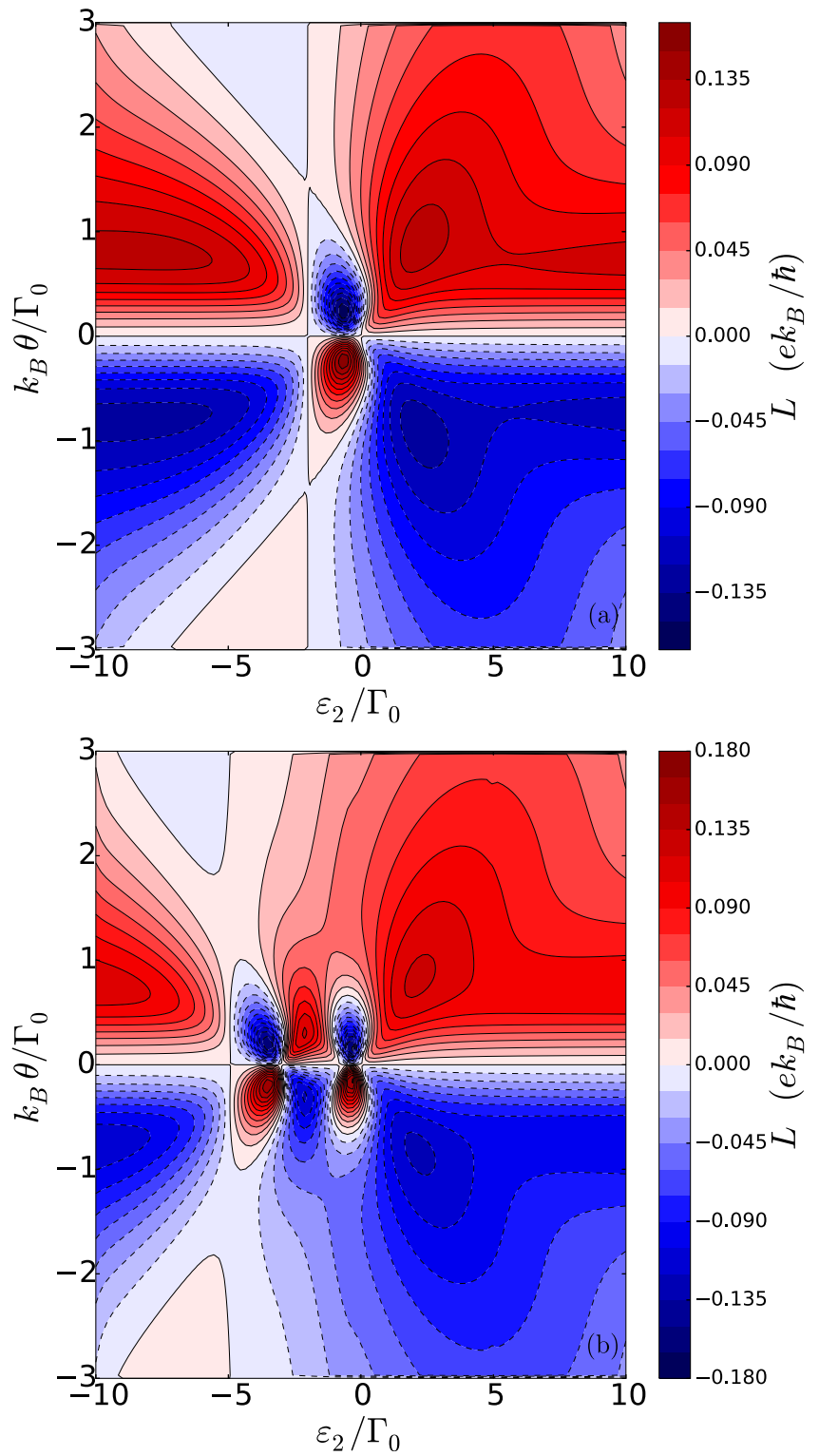

FIG. 6. Differential thermoelectric conductance as a function of the temperature bias $k_{B} \theta$ and the level position $\varepsilon_{2}$ for $\varepsilon_{1}=2$. (a) $U_{1}=$ $U_{2}=0$ (noninteracting case). (b) $U_{1}=U_{2}=3$ (interacting case). The background temperature is $k_{B} T=0.001$. Energies are expressed in units of $\Gamma_{0}$.

becomes asymmetric with two peaks in $\varepsilon_{1}$ and $\varepsilon_{2}$. Since $\varepsilon_{2}$ is closer to $\varepsilon_{F}$, the thermocurrent will flow from the right electrode when the left electrode is hotter. Therefore, $L$ is negative, as shown in the blue central area of Fig. 6(a). Further increase of $\varepsilon_{2}$ favors the transport of thermally excited electrons above the Fermi energy and $L$ turns out to be positive [the red region to the right of Fig. 6(a)]. For negative $\theta$, the thermoelectric conductance changes sign because the right contact is now hotter. The interacting case is plotted in Fig. 6(b). Based on the results depicted in Fig. 3(b), the transmission comprises multiple peaks that give rise to additional changes of sign for $L$ [compare Figs. 6(b) and 6(a)]. For example, $L$ is almost zero not only for $\varepsilon_{2}=-2 \Gamma_{0}$, but also now for $\varepsilon_{2}=-5 \Gamma_{0}$. Nevertheless, we note that in the

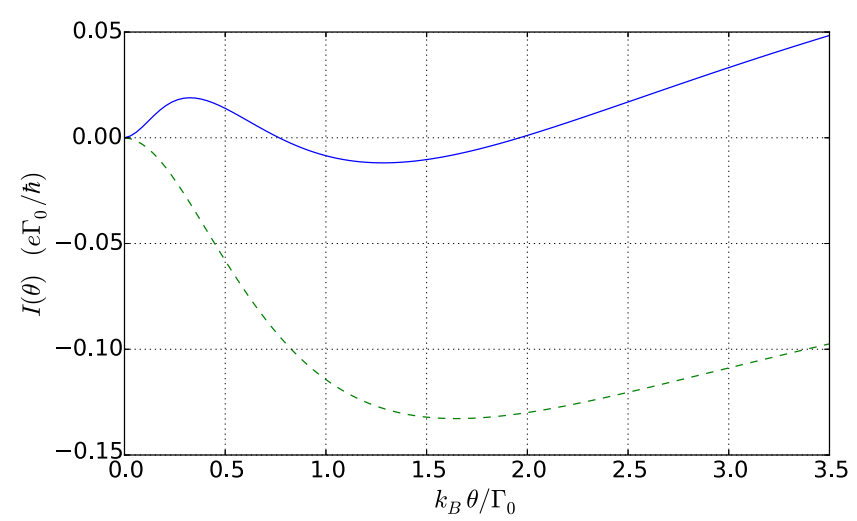

FIG. 7. Thermocurrent as a function of the temperature bias $k_{B} \theta$ for different values of the charging energy. Blue curve: $U_{1}=U_{2}=$ 1.2; green curve: $U_{1}=U_{2}=0$. The energy level positions of the dot are $\varepsilon_{1}=4.0$ and $\varepsilon_{2}=-1.05$. The background temperature is $k_{B} T=0.001$. Energies are expressed in units of $\Gamma_{0}$.

interacting situation, the symmetry of the transmission line shape is only approximate, unlike the noninteracting case. As a consequence, the thermoelectric conductance quickly becomes nonzero when $\theta$ departs from the linear regime.

The rich structure of the differential thermoelectric conductance discussed above suggests that the thermocurrent $I(\theta)$ might cross the $\theta$ axis several times unlike the single dot case, where there exists one nontrivial zero only [49-57]. In Fig. 7, we show a representative result of $I(\theta)$ for different values of the charging energy. Remarkably, the thermocurrent becomes strongly nonlinear and changes sign twice for a tuning of the dot levels such that $\varepsilon_{1}=4$ and $\varepsilon_{2}=-1.05$ (blue curve). For comparison, we also show the noninteracting case (green curve) for which the sign of I stays the same. Strikingly enough, the current-temperature curve when interactions are present presents a marked region of negative differential conductance reminiscent of the current-voltage characteristics of Esaki diodes [58]. The difference is that in our case, transport is driven purely by thermal means. We obtain a peak-to-valley ratio (defined as the distance between the maximum and the minimum magnitudes divided by the current peak) of the order of 1.7.

Since the existence of negative differential conductance driven by a temperature gradient is an important result, we explain the origin of the current behavior in the sketch of Fig. 8. In Fig. 8(a), we plot the transmission probability as a function of energy calculated for the nonzero value of a small thermal bias. This curve has to be convoluted with the Fermi function difference [see Fig. 8(b)] in order to calculate the full thermoelectric current $I$ given by Eqs. (2a) and (11). For a given $\theta, f_{L}-f_{R}$ is an antisymmetric function of $E$ which is nonzero for energies smaller than or of the order of $\pm 3 k_{B} \theta$. Note that the difference is positive (negative) for energies above (below) the Fermi energy $\varepsilon_{F}=0$, as it should be. When multiplied by the transmission, this means that for $\theta=0.20$, the contribution from the electrons (carriers traveling above $\varepsilon_{F}$ ) is larger (red color) than that from the holes (carriers traveling below $\varepsilon_{F}$, blue color). As a consequence, the net current is positive (see the blue curve of Fig. 7 for $\theta=0.20$, i.e., before the first nontrivial zero). When 

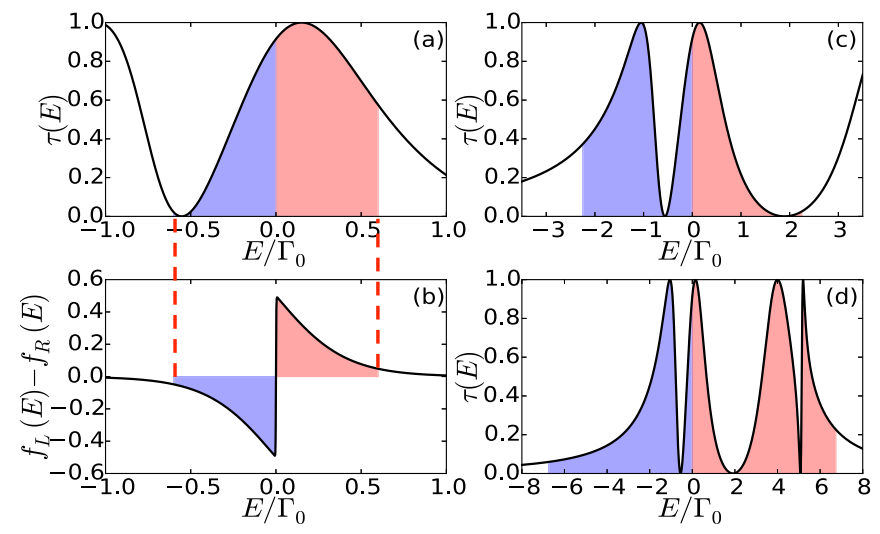

FIG. 8. Sketch explaining the nonlinear behavior of the thermocurrent. Transmission function as a function of energy for (a) $\theta=0.2$, (c) $\theta=0.75$, and (d) $\theta=2.25$. Charging energies are $U_{1}=U_{2}=1.2$ and background temperature is $k_{B} T=0.0001$ (in units of $\Gamma_{0}$ ). The energy level positions of the dot are $\varepsilon_{1}=4.0$ and $\varepsilon_{2}=-1.05$. The color filling represents the part of the transmission function which contributes to the transport at given $\theta$. These parts are calculated from the overlap with the Fermi function difference [panel (b) for $\theta=0.2]$ which is nonzero within the energy range indicated with vertical dashed lines. Blue (red) filling represents transport of electrons (holes).

the temperature bias increases, the transmission is modified accordingly [see Fig. 8(c) for $\theta=0.75$ ]. In this case, the hole-type transport surpasses the flow of electrons because the energy range for $f_{L}-f_{R}$ has to be extended (the occurrence of an antiresonance in $\tau$ also plays a role). Therefore, $I$ becomes negative and a nontrivial zero emerges at around $\theta=0.7$. Further enhancement of $\theta$ involves an even larger energy interval, which now covers additional transmission peaks at higher energies [see Fig. 8(d) for $\theta=2.25$ ]. Then, the electron current again becomes dominant and $I$ attains a positive value, which implies a second nontrivial zero at around $\theta=2.0 \mathrm{in}$ Fig. 7.

\section{CONCLUSIONS}

In summary, we have analyzed the impact of electronelectron interactions in the spectral density of a parallel DQD system, highlighting the role of BICs. We find that the existence of these states is a robust phenomenon, offering the opportunity of exploiting it in electric and thermoelectric nanodevices. To show this, we have discussed the changes in the Coulomb diamond structure of the differential conductance and the presence of maxima and minima in the energy diagram that can be detected in a transport experiment. More importantly, the thermoelectric conductance exhibits a series of asymmetric peaks when the Fermi energy is varied across the system resonances. Maps of the thermoelectric response show a distorted butterfly structure due to intradot charging effects. In the nonlinear regime of transport, we have found nontrivial zeros and negative differential conductances as a function of the applied thermal gradient, which opens up the possibility of employing parallel double dots as nanoscale amplifiers and generators of electronic oscillations based on thermal gradients only.

\section{ACKNOWLEDGMENTS}

We thank R. López for useful discussions. This work has been supported by MINECO under Grants No. FIS201452564, No. MAT2013-46308, CAIB, and FEDER. M.S-B. and F.D-A. thank the Theoretical Physics Group of the University of Warwick for the warm hospitality.
[1] A. W. Holleitner, C. R. Decker, H. Qin, K. Eberl, and R. H. Blick, Phys. Rev. Lett. 87, 256802 (2001).

[2] A. W. Holleitner, R. H. Blick, A. K. Hüttel, K. Eberl, and J. P. Kotthaus, Science 297, 70 (2002).

[3] J. C. Chen, A. M. Chang, and M. R. Melloch, Phys. Rev. Lett. 92, 176801 (2004).

[4] M. L. Ladrón de Guevara, F. Claro, and P. A. Orellana, Phys. Rev. B 67, 195335 (2003).

[5] H. Lu, R. Lü, and B.-f. Zhu, Phys. Rev. B 71, 235320 (2005).

[6] P. Trocha and J. Barnaś, Phys. Rev. B 76, 165432 (2007).

[7] P. A. Orellana, M. L. Ladrón de Guevara, and F. Claro, Phys. Rev. B 70, 233315 (2004).

[8] J. König and Y. Gefen, Phys. Rev. B 65, 045316 (2002).

[9] G.-H. Ding, C. K. Kim, and K. Nahm, Phys. Rev. B 71, 205313 (2005).

[10] Y. Tanaka and N. Kawakami, Phys. Rev. B 72, 085304 (2005).

[11] H. Lu, R. L, and B. f. Zhu, J. Phys.: Condens. Matter 18, 8961 (2006).

[12] R. López, R. Aguado, and G. Platero, Phys. Rev. Lett. 89, 136802 (2002).

[13] D. Krychowski and S. Lipiński, Phys. Rev. B 93, 075416 (2016).

[14] V. I. Litvinov, Phys. Rev. B 76, 245305 (2007).
[15] R. López, D. Sánchez, M. Lee, M.-S. Choi, P. Simon, and K. Le Hur, Phys. Rev. B 71, 115312 (2005).

[16] A. Wong, W. B. Lane, L. G. G. V. Dias da Silva, K. Ingersent, N. Sandler, and S. E. Ulloa, Phys. Rev. B 85, 115316 (2012).

[17] D. Loss and E. V. Sukhorukov, Phys. Rev. Lett. 84, 1035 (2000).

[18] A. Ramšak, J. Mravlje, R. Žitko, and J. Bonča, Phys. Rev. B 74, 241305 (2006).

[19] U. Fano, Phys. Rev. 124, 1866 (1961).

[20] A. E. Miroshnichenko, S. Flach, and Y. S. Kivshar, Rev. Mod. Phys. 82, 2257 (2010).

[21] J. von Neumann and E. Wigner, Phys. Z. 30, 465 (1929).

[22] C. González-Santander, P. A. Orellana, and F. DomínguezAdame, Europhys. Lett. 102, 17012 (2013).

[23] C. Álvarez, F. Domínguez-Adame, P. A. Orellana, and E. Díaz, Phys. Lett. A 379, 1062 (2015).

[24] Y. S. Liu and X. F. Yang, J. Appl. Phys. 108, 023710 (2010).

[25] P. Trocha and J. Barnaś, Phys. Rev. B 85, 085408 (2012).

[26] G. D. Mahan and J. O. Sofo, Proc. Natl. Acad. Sci. USA 93, 7436 (1996).

[27] H. J. Goldsmid, Introduction to Thermoelectricity (Springer, Berlin, 2010). 
[28] D. Sánchez and H. Linke, New J. Phys. 16, 110201 (2014).

[29] L. D. Hicks and M. S. Dresselhaus, Phys. Rev. B 47, 16631 (1993).

[30] A. Khitun, A. Balandin, J. L. Liu, and K. L. Wang, J. Appl. Phys. 88, 696 (2000).

[31] A. A. Balandin and O. L. Lazarenkova, Appl. Phys. Lett. 82, 415 (2003)

[32] R. Venkatasubramanian, E. Siivola, T. Colpitts, and B. O'Quinn, Nature (London) 413, 597 (2001).

[33] T. C. Harman, P. J. Taylor, M. P. Walsh, and B. E. LaForge, Science 297, 2229 (2002).

[34] A. I. Hochbaum, R. Chen, R. Diaz Delgado, W. Liang, E. C. Garnett, M. Najarian, A. Majumdar, and P. Yan, Nature (London) 451, 163 (2008).

[35] A. I. Boukai, Y. Bunimovich, J. Tahir-Kheli, J.-K. Yu, W. A. Goddard, III, and J. R. Heath, Nature (London) 451, 168 (2008).

[36] J. P. Bergfield and C. A. Stafford, Nano Lett. 9, 3072 (2009).

[37] O. Karlström, H. Linke, G. Karlström, and A. Wacker, Phys. Rev. B 84, 113415 (2011).

[38] M. Saiz-Bretín, A. V. Malyshev, P. A. Orellana, and F. Domínguez-Adame, Phys. Rev. B 91, 085431 (2015).

[39] G. Gómez-Silva, O. Ávalos Ovando, M. L. Ladrón de Guevara, and P. A. Orellana, J. Appl. Phys. 111, 053704 (2012).

[40] K. P. Wójcik and I. Weymann, Phys. Rev. B 93, 085428 (2016).

[41] J. Zheng, M.-J. Zhu, and F. Chi, J. Low Temp. Phys. 166, 208 (2012).
[42] H. Haug and A. P. Jauho, Quantum Kinetics in Transport and Optics of Semiconductors (Springer, Berlin, 2008).

[43] A. C. Hewson, Phys. Rev. 144, 420 (1966).

[44] M. Bagheri Tagani and H. Rahimpour Soleimani, Phys. Scr. 86, 035706 (2012).

[45] Y. Meir and N. S. Wingreen, Phys. Rev. Lett. 68, 2512 (1992).

[46] B. R. Bułka and P. Stefański, Phys. Rev. Lett. 86, 5128 (2001).

[47] N. M. Gergs, C. B. M. Hörig, M. R. Wegewijs, and D. Schuricht, Phys. Rev. B 91, 201107 (2015).

[48] M. Cutler and N. F. Mott, Phys. Rev. 181, 1336 (1969).

[49] M. A. Sierra and D. Sánchez, Phys. Rev. B 90, 115313 (2014).

[50] A. Svilans, A. M. Burke, S. Fahlvik Svensson, M. Leijnse, and H. Linke, Physica E 82, 34 (2016).

[51] D. Sánchez and R. López, arXiv:1604.00855.

[52] S. F. Svensson, E. A. Hoffmann, N. Nakpathomkun, P. M. Wu, H. Q. Xu, H. A. Nilsson, D. Sánchez, V. Kashcheyevs, and H. Linke, New J. Phys. 15, 105011 (2013).

[53] J. Azema, P. Lombardo, and A.-M. Daré, Phys. Rev. B 90, 205437 (2014).

[54] S.-Y. Hwang, R. López, M. Lee, and D. Sánchez, Phys. Rev. B 90, 115301 (2014).

[55] N. A. Zimbovskaya, J. Chem. Phys. 142, 244310 (2015).

[56] A. E. Stanciu, G. A. Nemnes, and A. Manolescu, Rom. J. Phys. 60, 716 (2015).

[57] J.-H. Jiang, M. Kulkarni, D. Segal, and Y. Imry, Phys. Rev. B 92, 045309 (2015)

[58] L. Esaki, Phys. Rev. 109, 603 (1958). 